\title{
La epistemología del sujeto conocido y la concepción del trabajo en Colombia
}

Omar Cabrales Salazar*

Resumen. Este artículo expone el marco metodológico de la tesis doctoral del autor. Plantea en primer lugar, como antecedentes, los cambios generados en el trabajo en el mundo occidental, desde el estado del bienestar hasta nuestros días, haciendo énfasis en los años de la globalización y el neoliberalismo y teniendo como referentes entre otros autores a Rifkin (2004), De la Garza (2001), Beck (2000), Gorz (1982) y Offe (1985). De la misma forma, cómo eventos que podrían crear categorías sociohistóricas importantes en la historia del trabajo en Colombia, se mencionan las tres reformas laborales promovidas por la ley 50 de 1990, la Ley 100 de 1993 y la Ley 789 de 2002. Posteriormente se hace referencia a la epistemología del sujeto conocido (Vasilachis, 2006) como fundamentación epistemológica que guía el trabajo de campo.

Palabras clave. Epistemología del sujeto conocido, fin del trabajo, precarización laboral, conceptos del trabajo.

* Economista de la Universidad Militar Nueva Granada con Especialización en Pedagogía y Docencia Universitaria, Maestría en Educación de la Pontificia Universidad Javeriana y candidato a Doctor en Ciencias Sociales y Humanas de la Pontificia Universidad Javeriana de Bogotá. Correo: omcabrales @yahoo.com.ar 
Abstract. The paper is based on the methodological framework of the doctoral thesis of the author. Arises primarily as background changes caused at work in the Western world, from the welfare state to the present day, focusing on the years of globalization and neoliberalism and taking as references from other authors to Rifkin (2004), De la Garza (2001), Beck (2000), Gorz (1982) and Offe (1985). Likewise, as events that could create major socio-historical categories in the history of work in Colombia, mentioned the three labor reform promoted by the law 50 of 1990, Law 100 of 1993 and Law 789 of 2002. Subsequently referred to the epistemology of the subject known (Vasilachis, 2006) and epistemological foundation that guides the fieldwork.

Key Words. Epistemology of the subject known, end of work, job insecurity, works concepts.

\section{Introducción}

Esta investigación tuvo como objetivo indagar cuáles son las concepciones o representaciones sobre el trabajo que tienen hoy un grupo de colombianos de tres generaciones distintas y sucesivas cuyas vidas se hayan visto inmersas en diferentes sentidos e intensidades en la globalización y en las consecuencias que el neoliberalismo ha dejado en el trabajo. Un primer grupo focal constituido por personas nacidas en los años 30 y 40 del siglo XX, un segundo grupo constituido por personas nacidas en los años 60 y 70 del siglo pasado y un tercer grupo focal constituido por personas nacidas en los años 80 y 90 del siglo pasado. Esta categorización obedece a que sería interesante evidenciar lo que piensan sobre el trabajo personas que han estado sometidas o que han vivido en un menor y mayor grado la globalización y el neoliberalismo, pues es evidente que se han suscitado cambios en el trabajo a partir de la crisis de los años 70 y que hay una marcada diferencia entre el trabajo de las décadas de los años dorados del capitalismo y el 
trabajo de los últimos 25 años del siglo XX y la primera década del siglo XXI (Antunes, Pochmann; 2008). A partir de encuestas y entrevistas a grupos focales, se pretende indagar por su percepción sobre lo que significaba y significa el trabajo, las actividades a las que se denominaba y que ahora se denominan como tal, el valor que se le daba y se le da al mismo, los significados y la importancia que se le da como actividad diaria o cotidiana a partir de la cual hay que ganarse la vida, y las mutaciones que en estos sentidos se han dado en las tres generaciones mencionadas. Esto con el propósito de conocer de primera mano lo que tres generaciones de colombianos piensan sobre el trabajo y la forma como su concepción cambia y transmuta hacia otras esferas y actividades, de manera que nos permita entender y plantear desde su percepción algunas sugerencias a las políticas públicas contra el desempleo y la posibilidad de emplearse de las generaciones siguientes.

Partiendo de las crisis y los cambios que se han dado al respecto de la función o el sentido del trabajo en los últimos 30 años como consecuencia del neoliberalismo y la globalización (Rifkin, 2004), (De la Garza, 2001), (Beck, 2000), (Gorz,1982), (Offe, 1985), (Antunes, Pochmann; 2008), se plantean tres categorías de análisis para explorar lo que los dos primeros grupos focales pensaban sobre el trabajo antes del neoliberalismo y la globalización y lo que piensan hoy los tres grupos focales. Estas categorías son las siguientes: en primer lugar, la crisis de la actividad laboral como espacio de socialización y centro de la creación de sujetos sociales (Jaureguì, 1998), (Medá, 1995) o de la centralidad del trabajo en la sociedad posindustrial (Touraine, 1999). En segundo lugar, la crisis del trabajo o del empleo «normal» como forma de conseguir los recursos para el sustento o núcleo de la acumulación de capital o como medio para ganarse la vida y alcanzar el objetivo de la abundancia (Gorz, 1991), (Pérez, 1997), (De la Garza, 1999), que nos conducen a explorar las categorías del desempleo, subempleo, empleo marginal y precarización laboral, entre otros. Y finalmente, la crisis del trabajo como fuente de la autorrealización personal y

ITINERARIO EDUCATIVO • AÑO XXVI, N. ${ }^{\circ} 59 \cdot 53-78$ • ENERO - JUNIO DE ZOIS 
constructor de la identidad (Offe, 1985). Otras categorías podrían surgir en la medida que avance la investigación y se evidencien otras percepciones sobre cómo ha cambiado el trabajo en Colombia, desde el punto de vista de estas tres generaciones, o cómo intergeneracionalmente han cambiado ellas en relación con lo que ha ocurrido en el mundo del trabajo con el neoliberalismo y la globalización.

Para tales efectos se reconstruirá el concepto de trabajo desde el marco teórico, y el que resulte de la percepción de los grupos focales siguiendo los principios de la epistemología del sujeto conocido de Vasilachis (2006) y bajo el enfoque de la investigación cualitativa, que por ser considerada como multimetódica, naturalista e interpretativa (Denzin y Lincoln, 1994) es la que más se adapta a los objetivos de la investigación, puesto que los investigadores cualitativos indagan en situaciones naturales, intentando dar sentido o interpretar los fenómenos en los términos del significado que las personas le otorgan (Denzin y Lincoln, 1994).

\section{Antecedentes}

No siempre se le ha dado el mismo sentido o la misma significancia al concepto de trabajo. Concebido desde la Modernidad como la actividad llevada a cabo para ganarse el sustento, lo que entendemos por trabajo en la época contemporánea es el resultado de una construcción conceptual que empezó a elaborase en el siglo XVIII, que se transformó en el siglo XIX y se consolidó en el siglo XX (Gorz, 1991), y que al día de hoy, en el siglo XXI está permanentemente sujeta a mutaciones profundas que repercuten esencialmente en la vida del hombre, fundamentalmente en su concepción sobre la calidad y el sentido de la vida.

Es un hecho que desde hace escasamente dos siglos nuestras sociedades se basan en el trabajo. Esto significa que en ellas el trabajo reconocido como tal, es decir, remunerado, se ha convertido en el 
principal medio para costear la subsistencia individual, pero también se ha constituido en una relación social de carácter fundamental, y, finalmente, representa la vía, nunca puesta en duda mediante la cual se alcanza el objetivo de la abundancia (Medá,1995).

Ahora bien, la significación que ha tenido el trabajo a lo largo de la historia universal, está referida a los diferentes enfoques que dan forma a la producción y a los modelos económicos que en últimas han constituido el andamiaje social y económico en el que se estructura la civilización occidental. El momento en el que el trabajo pasa de ser la labor cotidiana de ejercer el oficio a través del cual el individuo se forjaba a sí mismo de acuerdo a su profesión: herrero, carpintero, pastor, agricultor, y se convierte en la labor de masas de trabajadores remunerados, se fija en el siglo XVIII, cuando se dejó de trabajar para sí o para el núcleo familiar y se trabajó para otro, es decir, el momento en el que el trabajo abandona la motivación primigenia y el sentido de la vida humana, y nace la figura del patrono; el momento en el que el trabajo se convierte en una mercancía cuantificable que alguien está dispuesto u obligado a trocar o a vender y otro dispuesto a comprarlo o a intercambiarlo. Adam Smith, es el primero en cuantificar la labor en términos monetarios, dentro de su propósito de explicar la riqueza de las naciones.

Smith introduce así, seguramente sin ser consciente de ello, una nueva definición del trabajo. Una definición que nos es fruto de un estudio de la característica compartida por las distintas formas concretas, prácticas del trabajo, sino que resulta de una investigación que no tiene realmente por objeto el trabajo; una investigación al término de la cual, el trabajo aparece como un instrumento de cálculo y medida, como instrumento cuya cualidad esencial es permitir el intercambio. (Medá, 1995).

En el caso colombiano, se afirma entonces que las sociedades conquistadas por los españoles, fundamentalmente la Nueva 
Granada, son el resultado de un proceso sincrético en el que se mezclan grupos étnicos de los tres continentes involucrados: África, Europa y América, dando como resultado una riqueza cultural y unas formas particulares de asumir la ciudadanía, el trabajo, las formas de pensar, ser y estar en el mundo, que ha estado ligada a factores como el tipo de individuo que llegó de la península Ibérica, la religión que profesaba y la forma en que pone en marcha la explotación y exportación del oro y la plata (Yunis, 2004). Estas circunstancias pudieron haber generado el mito de que existe una tendencia del colombiano a rehuirle al trabajo duro y a buscar el enriquecimiento rápido y fácil, que se convierte en un factor generador de permanente ilegalidad y violencia, o por el contrario, ha hecho de los colombianos unos trabajadores hacendosos, recursivos y creativos, dispuestos a aguantar extensas jornadas laborales y a buscarse el sustento a como dé lugar.

A finales del siglo $X V$, con el surgimiento del capitalismo mercantil se abren más posibilidades de comercio y por ende de enriquecimiento de los europeos. En un primer sentido, por el incremento de transacciones comerciales inherente al crecimiento del capitalismo, debido, entre otras cosas, al cambio de la concepción de la riqueza de la tierra hacia el dinero. Como lo afirma Tirado (1981): «Con el crecimiento del comercio y la reaparición de la circulación monetaria, la tierra dejó de ser la fuente principal de la riqueza y la base del poder político». Esta nueva percepción de la riqueza, que ya no es sólo accesible para los nobles sino para otros astutos mortales a partir del ejercicio del trabajo, se consolidará con la Reforma y será la semilla en esencia del capitalismo del siglo XVIII, puesto que cuando el dinero adquiere otra función además de la de equiparar valor y permitir las transacciones, y se convierte en sí mismo en fuente generadora de riqueza; el afán de atesorar se acentuará en los hombres y los conducirá a trabajar, a emprender el viaje a América o a delinquir para adquirir lo que, por no tener tierra ni títulos nobiliarios, les permitirá asemejarse a la nobleza y empezar a constituir de una nueva clase social: la burguesía. A 
mediados del siglo XVIII se produce como dice Medá una brusca inversión de valores que centra en la riqueza el verdadero propósito que deben perseguir las sociedades.

Se han propuesto muchas explicaciones de este momento histórico, el momento, en definitiva, de la fundación de la sociedad moderna. Algunas explicaciones ven la Revolución Industrial, especialmente en su vertiente técnica, el desencadenante, primero, del aumento de la productividad y, en consecuencia, del interés por la riqueza. (Méda,1995).

Antes de la Revolución Industrial, el sentido trascendente del trabajo era lo que realmente conectaba al hombre con su realidad, al punto de ser lo que su oficio le trazaba: herrero, carpintero, albañil, etc. Con la constitución de las cadenas de montaje que se empezaban a implementar en la fábrica, había entonces que revertir ese sentimiento de libertad del artesano hacia las cadenas de producción, de manera tal que las consecuencias trascendentes del trabajo se trasladaran al resultado de la explotación del operario. La ética del trabajo se encargó de este proceso y constituyó el trabajo desde la Revolución Industrial como parte fundamental de la vida, al punto de convertirlo en un instrumento de explotación del trabajador por parte del capitalista.

El trabajo entonces se constituyó como parte de la condición humana. Según Hanna Arendt (1998) la sociedad occidental es una sociedad del trabajo, su paradigma fundamental, a partir de la Revolución Industrial, es el trabajo que produce. El trabajo, considerado como actividad recurrente, diaria e inobjetable a partir de la cual hay que ganarse la vida conformó entonces una sociedad de productores que asentó en el trabajo el sentido de la vida y la misma condición humana. «El trabajo expresaría, por tanto en el mayor grado nuestra humanidad, nuestra condición de seres finitos, creadores de valores, y también nuestra condición de seres sociales. El trabajo sería, pues, nuestra esencia y nuestra 
condición» (Medà, 1998). Es así como a partir de la Revolución Industrial que el trabajo empieza a constituir al propio hombre, lo erige en torno a sus significancias y circunstancias hasta hacer parte de su esencia, de su condición humana. El hombre, a partir de la Revolución Industrial es humano porque trabaja, el trabajador hace parte de la comunidad humana, el desempleado es excluido.

Fue entonces a partir de la Revolución Industrial, como resultado del proceso de masificación de la sociedad en torno al trabajo o la fábrica, que el hombre salió de su casa y dejó de practicar el oficio que había heredado de sus antepasados. El hecho de salir de su lugar de habitación, que era también su lugar de trabajo, determinó un cambio en su condición como ser humano puesto que su identidad como carpintero o herrero, se diluía en la conflagración de los intereses del proletariado. «Al igual que el molino de papel de Diderot, la fábrica de Smith es un lugar para trabajar, no para vivir. La separación de la casa y el trabajo, es según Smith, la más importante de todas las divisiones modernas del trabajo» (Sennett, 2006). Vemos entonces una distinción clave en el proceso de conceptualización del trabajo, al pasar de la palabra oficio, entendida como la labor que se lleva cabo para ganarse el sustento en el lugar de habitación, en la casa y por lo general heredada de los padres y abuelos, a la palabra trabajo como actividad llevada a cabo con el mismo propósito pero fuera de la casa. Este hecho fundamental crea el concepto de trabajadores, al unificar, no solo las labores propias del proceso de producción de la fábrica, sino la propia percepción e interpretación del mundo.

Marx será el primero en denunciar el nuevo afán de lucro del capitalista a partir de la explotación del trabajador. «La principal crítica de Marx consiste en denunciar que la sociedad de su tiempo y la economía política no tiene como finalidad fomentar el desarrollo del hombre mediante el trabajo -por esencia mediador- sino el enriquecimiento» (Medá, 1995). Desde la perspectiva marxista, las relaciones sociales de producción de una sociedad constitu- 
yen la base de su estructura de clases. La posesión por parte del capitalista de la propiedad privada de los medios de producción es la causa fundamental de la desigualdad dentro de la sociedad capitalista. De hecho, la desigualdad es inherente al capitalismo. En este sentido, Marx afirma,

decir que los intereses del capital y los intereses de los obreros son los mismos equivale simplemente a decir que el capital y el trabajo asalariado son dos aspectos de una misma relación. El uno se halla condicionado por el otro, como el usurero por el derrochador y viceversa (...) Incluso la situación más favorable para la clase obrera, el incremento más rápido posible del capital, por mucho que mejore la vida material del obrero, no suprime el antagonismo entre sus intereses y los intereses del burgués, los intereses del capitalista. Ganancia y salario seguirán hallándose, exactamente lo mismo que antes, en razón inversa.

El trabajador entonces «es menos todavía que una bestia de carga. Físicamente destrozado y espiritualmente embrutecido, es una simple máquina para producir riqueza ajena». (Marx, Engels: 1865, 225).

En términos generales, la sociedad del trabajo se constituye con la Revolución Industrial, todas sus acepciones complejas al respecto de la explotación del trabajador y de su connotación reivindicativa de la vida se van fraguando durante este periodo y nos darán luces a la hora de aplicar el concepto en la constitución histórica del trabajo en Colombia y la percepción que se tiene al respecto de su valor hoy en día.

Bauman $(2000,48)$, resalta la sociedad de consumo frente a la sociedad de productores, puesto que en la etapa industrial de la modernidad había un hecho incuestionable: antes que cualquier cosa todos debían ser ante todo productores. En esta segunda modernidad o posmodernidad la primera imperiosa obligación es ser consumidor. 
La forma en que esta sociedad moldea a sus integrantes está regida ante todo y en primer lugar; por la necesidad de desempeñar ese papel; la norma que les impone, la de tener capacidad y voluntad de consumir (Bauman, 2000, 44). La metamorfosis del concepto de consumo desde el vicio hasta la virtud es uno de los fenómenos más importantes observados durante el transcurso del siglo XX (Rifkin, 2004, 71). De hecho, los trabajadores de principios del siglo pasado se conformaban con ganar lo justo para vivir y para darse algunos lujos elementales, y preferían tener más tiempo de ocio en lugar de ingresos adicionales que resultaran de una mayor cantidad de horas de trabajo. Este aspecto se convirtió en una preocupación para los hombres de negocios de la época cuyos inventarios se amontonaban rápidamente en sus fábricas y en sus almacenes por toda Norteamérica.

Se propuso entonces cambiar la psicología del trabajador productor ahorrador hacia una del trabajador consumidor y se creo la figura del «consumidor insatisfecho»; acompañada de algunas otras estrategias como la difusión del concepto de la moda, el estatus, y la preferencia hacia los productos de tienda con una marca que empezaba a consolidarse, frente a los productos caseros.

La ética protestante del trabajo, que había dominando el comportamiento del americano de frontera, estaba profundamente enraizada en el comportamiento general. La moderación y el sentido del ahorro eran piedras angulares en el estilo de vida americano (...) La comunidad empresarial americana se propuso cambiar radicalmente la psicología que había construido una nación -su objetivo era convertir a los trabajadores americanos desde la postura de inversores en el futuro a la de consumidores en el presente. (Rifkin, 2004, 72).

Consolidada la sociedad de consumo con la televisión y los electrodomésticos después de la Segunda Guerra Mundial, el gasto del gobierno amainaba un poco el impacto del desempleo de los 
trabajadores sustituidos por las máquinas. «En Estados Unidos, en 1929 el gasto gubernamental era tan solo del 12\% del PIB. En 1975 el gasto total había crecido hasta una cifra alrededor del 33\% del PIB». (Alperovitz, 1993, 20). El sector de servicios contribuyó también a que el impacto no fuera tan grave.

La idea de Estado benefactor mantiene una relación ambigua con la ética del trabajo. Por un lado, los partidarios de garantizar colectivamente el bienestar individual reconocieron siempre el carácter normal de una vida sostenida por el trabajo; señalaban, sin embargo, que la norma no era válida universalmente debido a que no todos lograban un empleo permanente. (Bauman, 2000, 64). En este sentido, el Estado benefactor debía proveer de puestos de trabajo a la sociedad para mantener la ética del trabajo, y como medida de salud social, creando entonces, además de una dependencia económica una dependencia política. Transformaba el derecho a una vida digna en cuestión de ciudadanía política, ya no de desempeño económico (Bauman, 2000, 74).

Hoy en día, después de más de 30 años de globalización, la situación en el mundo es más que preocupante. La crisis económica de los años setenta generada por la guerra de Jom Ki Pur, y el consecuente incremento de los precios del petróleo, entre otras causas, sentó las bases para que las propuestas de Hayek y Friedman tomaran vuelo y se empezaran a implementar en Chile, Inglaterra y Estados Unidos: el neoliberalismo encontraba las bases de su justificación.

Bajo este modelo el concepto de «crecimiento económico» va unido al reemplazo de puestos de trabajo estables por "mano de obra» flexible, a la sustitución de la seguridad laboral por contratos renovables, en últimas; las reestructuraciones y la racionalización implican reducciones de personal. El éxito de los ejecutivos de las multinacionales está ligado a la maximización de las utilidades, tomando medidas en contra del empleo y de la estabilidad de los 
trabajadores, inclusive con la supresión de miles de puestos de trabajo: a medida que los pobres se hacen más pobres, los ricos -dechados de virtudes para la sociedad de consumo- se vuelven más ricos todavía (Bauman, 2000, 69). El trabajo escasea porque la sociedad posmoderna ha permitido la disminución de los costos y la maximización de las ganancias, la implementación de las máquinas y de la tecnología en los sistemas productivos han desplazado la hora hombre, al punto de no necesitarlo. Como lo afirma Appadurai (2003), lo más grave es que antes los viejos ricos necesitaban a los pobres para hacerse y mantenerse ricos, pero ahora ni siquiera tienen necesidad de ellos, puesto que ya no hay necesidad de mano de obra más barata.

La economía global, sustentada en un gran porcentaje en las empresas multinacionales, quienes se han convertido en el principal motor de crecimiento frente a la disminución del tamaño del Estado, son las que tienen en gran parte el destino de la humanidad. Lastimosamente, los intereses de estas compañías en el modelo capitalista neoliberal, no comprenden ninguna consideración con el planeta, con el mismo Estado y con la clase trabajadora. Como lo afirma Beck (2008) el incremento de la productividad de estas compañías está ligado con la desaparición de puestos de trabajo, la productividad obligada de las multinacionales y la incapacidad de los gobiernos para generar nuevos puestos de trabajo. Pero aun así, si no hay trabajo no hay quien compre, y de alguna forma las economías del siglo XXI se han mantenido a flote empleando millones de trabajadores en lugares donde pueden pagar salarios de subsistencia.

A la problemática del desempleo, hay que sumar el hecho de que cada día más jóvenes ingresan al mercado laboral y el sistema económico mundial no está en capacidad de ofrecerles la posibilidad de emplearse. En este sentido, es importante para la investigación conocer las percepciones sobre el trabajo de aquellas generaciones que siendo jóvenes durante el periodo de bienestar lograron insertarse en el mercado laboral y disfrutar de alguna 
forma de las 'prebendas' que les ofrecía su empleo y aquellos que no lo lograron. De la misma forma, aquellos jóvenes que en unas condiciones de flexibilización y neoliberalización del mercado han logrado insertarse en el mundo regular del trabajo y los que no lo han logrado. Esto podría indicarnos si las crisis o los cambios son los que determinan las percepciones o no.

De acuerdo con las cifras entregadas por la Organización Internacional del Trabajo (OIT), alrededor de 10,2 millones de jóvenes pasaron a engrosar las filas de los parados entre 2007 y 2009, la más fuerte alza registrada desde «al menos» 1991. El índice global de desempleo para este grupo poblacional se situó en 13,4\% durante el año pasado. La OIT calcula que en 2010 la tasa mundial de desocupación estará cercana al 7\%, lo que significa que al cierre de este año habrá aproximadamente 228 millones de personas sin empleo. Para Sparreboom (2010), este aumento del desempleo juvenil durante la crisis «no es desproporcionado», pues el problema viene de antes y tiene un «carácter estructural». (Diario Portafolio, enero 23 de 2010).

Adicionalmente, las diferentes crisis económicas que se han venido presentando desde la caída de la Unión Soviética han generado el cierre de grandes compañías con las consecuencias nefastas para sus empleados, o ajustes que redundan en la disminución de las nóminas de las empresas. El desempleo mundial causado por la última crisis del 2007 ha tenido efectos más nocivos en las economías industrializadas. De acuerdo con un informe de la OIT, la cantidad de personas sin trabajo se incrementó en 34 millones con respecto a 2007. Las diferencias regionales en las tasas de desocupación dan cuenta de que en los países desarrollados los efectos nocivos de la crisis se sintieron con más fuerza que en las economías emergentes. El informe revela, por ejemplo, que mientras en el este de Asia la tasa de desocupación terminó el año pasado en 4,4\%, en la Eurozona y Estados Unidos el índice cerró rondando el 10 por ciento. «Aunque representan menos del 16\% 
de la mano de obra mundial, estas regiones contribuyeron en más de un 40 por ciento al alza del desempleo mundial desde 2007», explica el estudio de la OIT. (Diario Portafolio, enero 23 de 2010). Estas circunstancias obligan a pensar en soluciones para ofrecerles a los jóvenes otras alternativas de vida que no estén enteramente ligadas al paradigma del trabajo como la única opción de vida o a la necesidad imperante de conseguir a toda costa un empleo remunerado. (Diario Portafolio, enero de 2010).

En Colombia, la apuesta de los últimos gobiernos por la reducción del tamaño del Estado por medio de la privatización y la delegación en el sector productivo de algunas de las responsabilidades que había adquirido durante el Estado del Bienestar, han generado un clima de incertidumbre laboral, la promoción de la desalarización, el autoempleo, el crecimiento del desempleo, del subempleo y del empleo informal, cuyas consecuencias son entre otras, la concentración de la riqueza, el empobrecimiento de una mayor parte de la población, el aumento de la inequidad y la pobreza.

No ajena a las exigencias del neoliberalismo, la legislación colombiana no se ha quedado atrás a la hora de promover las diferentes reformas que le han dado cabida a la ideología neoliberal. En primer lugar tenemos el Decreto 1433 de 1983, antecedente normativo de las Empresas de Servicios Temporales, en el cual se clarificó la naturaleza y características de la prestación del servicio temporal, siendo regulada legalmente con la reforma laboral de 1990. Este decreto comenzó a introducir a Colombia en el mundo de la tercerización y la flexibilización laboral con la contratación de trabajadores a través de las Empresas de Servicios Temporales, cuyos factores económicos y tecnológicos se sitúan en las raíces de los planteamientos flexibilizadores originados en los cambios que se dieron en Europa a partir de los años sesenta, al pasar de un importante crecimiento económico que venía desde la finalización de la Segunda Guerra Mundial, a un período de reestructuraciones industriales, irrupción de nuevas tecnologías, el surgimiento 
de nuevas potencias industriales (cuenca del Pacífico) y la crisis energética (Arenas, 1990). También hay factores de tipo ideológico en estos procesos que ya se han mencionado anteriormente como es el cuestionamiento al Estado del bienestar y el surgimiento de una mentalidad política con mayor énfasis en el mercado como mecanismo de asignación de recursos en la sociedad. Contexto en el que se delegó a las fuerzas del mercado y por ende a las multinacionales y no al gobierno a través de su política de regulación institucional, la regulación de la economía, incluyendo al propio mercado de trabajo. La protección laboral pasó a ser vista, entonces, como un elemento que impedía la competitividad y la inversión extranjera, por lo que se pasó a revisar la protección social y laboral existente (Bronstein, 1990). En relación a este tema se encuentran en general cuatro grandes vertientes de la flexibilidad laboral: la flexibilidad de los salarios, la flexibilidad de la duración del trabajo, la flexibilidad de la organización del trabajo y la flexibilidad de la contratación laboral, que se tratarán puntualmente en el momento que se lleve a cabo el análisis del Decreto 1433 de 1983 y de las reformas que modificaron estas circunstancias que en últimas resumen la necesidad de adaptar nuestra legislación laboral a las condiciones del mercado mundial.

Por otra parte tenemos Ley 50 de 1990, que dentro de la misma política normativa de ajustar la reglamentación interna al contexto neoliberal, pretendió en una primera instancia la reducción de los costos laborales al empresario para la generación de empleo. Como lo afirma Luis Jorge Garay (2004):

Determinar el impacto de la ley 50 sobre los costos laborales es una tarea compleja en la medida en que las modificaciones introducidas por esta ley se superponen con las normas introducidas con la reforma a la seguridad social, ley 100 de 1994, y la reforma al Servicio Nacional de Aprendizaje, que también afectaron los costos laborales de las empresas. Sin embargo, la sensación entre empresarios es que la relativa disminución de

ITINERARIO EDUCATIVO • AÑO XXVI, N. ${ }^{\circ} 59 \cdot 53-78$ • ENERO - JUNIO DE ZOIS 
los costos laborales proveniente de la reforma laboral, específicamente de la eliminación de la retroactividad de las cesantías y de la acción de reintegro, luego fueron rebasados por el aumento de las cotizaciones de la seguridad social a cargo de los empleadores, estipuladas en la respectiva reforma.

En términos generales, esta ley creó la figura del salario integral, incorporada en el Artículo 18 cuyo objetivo fue dar a conocer elementos sobre la compensación flexible y estableció una liquidación de las cesantías con base únicamente en el salario devengado, aunque se siguió reconociendo el $12 \%$ de intereses; sin embargo, este dinero fue destinado a los nuevos fondos de pensiones y cesantías, reduciendo el costo de las cesantías a 9,3 por ciento al año (Santa María, García, Mujica, 2009). Sus efectos son difíciles de prever en el mediano y largo plazo, por la superposición que se generó con la Ley 100. En cuanto a nuestro propósito, como es el de evidenciar en las generaciones de colombianos la percepción del trabajo antes y después de la citada ley, se espera, que a partir de indagación cualitativa centrada en la opinión del sujeto conocido, nos dé luces al respecto de las consecuencias que la citada ley generó en el trabajo en Colombia.

La Ley que continúa en nuestro estudio es la Ley 100 de 1993, la que reformó a la seguridad social en Colombia. Investigadores de Fedesarrollo concluyeron que el incremento de 14 puntos porcentuales en los costos no salariales, en un escenario donde la protección social se financia a través de impuestos a la nómina, ha generado una profunda segmentación del mercado laboral. Esta situación debe entenderse como la coexistencia de dos mercados laborales, uno asalariado y otro cuenta propia, que actúan como sustitutos el uno del otro. Por tanto, la reforma ha promovido la creación de empleos cuenta propia e informalidad, pues los trabajadores buscan emplearse en sectores donde pueden evadir estos pagos, mientras que las empresas se han visto desincentivadas a crear empleos formales. Este proceso ha sido particularmente 
nocivo para la población menos educada y más vulnerable, limitando así la generación de ingresos sostenibles y la superación de la pobreza (Santa María, García, Mujica, 2009). Las evidencias arrojan que se mejoró el acceso de la población vulnerable a los servicios de salud que ha redundado en una mejora general del estado de salud de la población colombiana. Sin embargo, estas mejoras han estado acompañadas de un aumento de 14 puntos porcentuales en los costos no salariales, lo cual ha afectado negativamente la evolución del mercado laboral. Se argumenta que estas contribuciones encarecen la generación de empleo (formal) lo que reduce, en equilibrio, el empleo, los salarios o ambos. También se reconoce que pueden conducir a un cambio en la composición del empleo, en el que los individuos prefieren buscar ocupaciones como el autoempleo o «cuenta propismo» en las que pueden evadir esos pagos (Santa María, García, Mujica, 2009). Como se puede ver, la reforma a la seguridad social ha promovido el subempleo y empleo informal y además ha evidenciado vínculos fuertes entre los esquemas de aseguramiento relacionados con la protección social y el mercado laboral.

Finalmente llegamos a la Ley 789 de 2002, que de la misma forma ha promovido la delegación en la sociedad civil de la seguridad social, la proliferación de contratos laborales temporales, la flexibilidad y la inestabilidad laboral, entre otras cosas. Como vemos, en términos generales la política laboral colombiana ha estado orientada a reducir los costos laborales como estrategia de generación de empleo y de crecimiento económico, como lo evidencia la última reforma laboral (Ley 789 de 2002) que entre otras cosas, redujo el pago por trabajo en días dominicales y festivos, amplió la jornada diurna y rebajó las indemnizaciones por despidos injustos.

\section{Metodología}

Según Vera (2004) la investigación cualitativa es aquella en la que se estudia la calidad de las actividades, relaciones, asuntos,

ITINERARIO EDUCATIVO • AÑO XXVI, N. ${ }^{\circ} 59 \cdot 53-78$ • ENERO - JUNIO DE ZOIL 
medios, materiales o instrumentos en una determinada situación o problema. La misma procura por lograr una descripción holística, esto es, que intenta analizar exhaustivamente todo el fenómeno en su conjunto y con sumo detalle. A diferencia de los estudios descriptivos, correlaciónales o experimentales, más que determinar la relación de causa y efecto entre dos o más variables, la investigación cualitativa se interesa más en saber cómo se da la dinámica o cómo ocurre el proceso en que se forma el asunto o problema. Asimismo, la investigación cualitativa permite familiarizarse con la forma como el grupo o persona experimenta, define y significa su realidad personal, interpersonal o cultural en el contexto de la vida cotidiana traduciéndolo en unas formas de pensar, actuar y sentir idiosincráticas o características (Quintana, 2006). En este sentido, este paradigma se aproxima a los intereses de esta investigación como son, explorar el concepto que tienen hoy del trabajo un grupo de colombianos de diferentes generaciones, puesto que implica acercarse al objeto de estudio, a un grupo humano, y escudriñar sus percepciones e intereses sobre el trabajo. Flick (1998), nos ayuda a consolidar dos de los rasgos más importantes sobre la investigación cualitativa.

La adecuación de los métodos y las teorías: el objetivo de la investigación es más descubrir lo nuevo y desarrollar teorías fundamentadas empíricamente que verificar teorías ya conocidas. La validez de la investigación se evalúa con referencia a aquello que se quiere estudiar y no depende exclusivamente del seguimiento de los abstractos criterios de la ciencia. La consigna central de la investigación cualitativa reposa en el origen de los resultados, en el material empírico y en la apropiada elección y aplicación de métodos al objeto de estudio.

Como se puede ver, los rasgos planteados por Flick (1998), junto con la propuesta de Vasilachis (2006), justifican en un amplio sentido la aplicación de la investigación cualitativa, puesto que 
se impone crear el concepto de trabajo desde la experiencia vital de algunos trabajadores y pensionados.

La perspectiva de los participantes y su diversidad: la investigación cualitativa analiza el conocimiento de los actores sociales y sus prácticas y tiene en cuenta que, en el terreno, los puntos de vista y las prácticas son distintos debido a los diferentes perspectivas subjetivas y a los disímiles conocimientos sociales vinculados con ellas (Flick, 1998).

Entonces, la metodología que seguirá la investigación es fundamentalmente de tipo cualitativo, aunque se diseñarán y aplicarán encuestas a los tres grupos de estudio. Ya que se pretende indagar por la percepción que tienen unos grupos sociales sobre un fenómeno complejo y dinámico como lo es el trabajo, la metodología cualitativa es conveniente para el logro del objetivo, puesto que según Mason (2006), la particularidad solidez de la investigación cualitativa yace en el conocimiento que proporciona acerca de la dinámica de los procesos sociales, del cambio y del contexto. Es así como a los tres grupos focales se les harán entrevistas para indagar sobre su concepción del trabajo, y se les aplicarán encuestas para obtener resultados manipulables sobre sus condiciones demográficas y tener la posibilidad de cruzar variables como por ejemplo la edad a la que empezaron a trabajar, las veces que lo han intentado y no lo han logrado, el tiempo que han estado trabajando, el nivel de ingresos, etc.

Los tres grupos focales están constituidos en primer lugar por personas nacidas en los años 30 y 40 del siglo XX, cuya infancia, adolescencia y adultez no se haya visto influenciada mayoritariamente por la globalización. Hago referencia a un grupo poblacional que al día de hoy tiene más de 60 años y que puede dar cuenta de cómo era el trabajo durante el periodo del estado del bienestar, pues se insertaron en el mundo del trabajo en los años 60 y 70 del siglo XX. En segundo lugar un grupo constituido por personas 
nacidas en los años 60 y 70 del siglo pasado, cuya niñez y adolescencia no hayan transcurrido en el periodo de la globalización, pero que ya en su vida adulta la hayan podido vivir, y un tercer grupo focal constituido por personas nacidas en los años 80 y 90 del siglo pasado cuya niñez y adolescencia hayan transcurrido en el periodo de la globalización. Estos grupos focales nos brindarán información relevante sobre lo que consideran como trabajo, para ir construyendo este concepto según la epistemología del sujeto conocido de Irene Vasilachis. Esta categorización obedece a que es evidente que se han suscitado cambios en el trabajo a partir de la crisis de los años 70 y que hay una marcada diferencia entre el trabajo de las décadas de los años dorados del capitalismo y el trabajo de los últimos 25 años del siglo XX (Antunes, Pochmann; 2008). Asimismo, estos cambios coinciden con las tres reformas laborales objeto de estudio: la Ley 50 de 1990, la Ley 100 de 1993 y la Ley 789 de 2002, considerando que la primera es el primer atisbo de la incidencia del neoliberalismo en la legislación colombiana que pretendía de alguna forma motivar el crecimiento del empleo y la reducción del desempleo ante las consecuencias económicas del fin del estado del bienestar.

En términos generales, la información demográfica y la que constituirá las variables a estudiar, como tasas de desempleo, tiempo y fuentes utilizadas para conseguir empleo, niveles de ingreso, etc., será obtenida por medio de métodos cuantitativos. La concepción sobre el trabajo se logrará por entrevistas a los grupos focales ya que la investigación cualitativa abarca el estudio, uso y recolección de una variedad de materiales empíricos -estudio de caso, experiencia personal, introspectiva, historia de vida, entrevista, textos observacionales, históricos, interaccionales y visuales-que describen los momentos habituales y problemáticos y los significados en la vida de los individuos (Vasilachis, 2003).

El grupo focal es la técnica de investigación cualitativa que indaga sobre las actitudes, intereses, opiniones y factores que motivan a 
un grupo homogenizado de personas-clientes. Korman, citado por Aigneren (2007) define los grupos focales como una reunión de un grupo de individuos seleccionados por los investigadores para discutir y elaborar, desde la experiencia personal, una temática o hecho social que es objeto de investigación. La técnica de los grupos focales se ha convertido en uno de los principales instrumentos de los métodos de indagación rápida desarrollados para obtener información ágil que posibilite dar respuesta en el corto plazo (Bonilla-Rodríguez, 1997).

Ahora bien, la epistemología del sujeto conocido que propone Vasilachis, hace referencia a otra forma de conocer y no excluye, sino que se complementa con la Epistemología del Sujeto Cognoscente en la que ubicamos a los paradigmas objetivistas. La epistemología se interroga acerca de cómo la realidad puede ser conocida, acerca de la relación entre quien conoce y aquello que es conocido, acerca de las características, de los fundamentos, de los presupuestos que orientan el proceso de conocimiento y la obtención de los resultados, acerca de la posibilidad de que ese proceso pueda ser compartido y reiterado por otros a fin de evaluar la calidad de la investigación y la confiabilidad de esos resultados (Vasilachis, 2003). De esta forma, la epistemología del sujeto conocido reconoce al sujeto en su calidad de ser humano como interlocutor válido, en su esencia, y en este sentido puede aportar desde su perspectiva del mundo de la vida a la construcción del concepto sobre el trabajo que se requiere en la investigación. Según Vasilachis (2006) la identidad del ser humano posee dos componentes, el esencial y el existencial.

Mientras el primero constituye el elemento común que identifica a los hombres/mujeres como hombres/mujeres y los iguala a los otros hombres/mujeres, el segundo constituye el aspecto diferencial que distingue a cada hombre/mujer de los otros y lo hace único/a frente a todos ellos. Así, por ejemplo, en un contexto espacial y temporalmente determinado, la identidad 
social, la política, la religiosa, la laboral serían expresiones del componente existencial de la identidad. Vasilachis (2006).

Habría que añadir, que lo que la autora argentina propone es que los dos marcos de interpretación, el del investigador (sujeto cognoscente) y el del investigado (sujeto conocido), generen una metaepistemología que contenga las dos epistemologías. Esta propuesta tiende a evitar que sean rechazadas otras formas de conocer distintas a las legitimadas actualmente en el campo de la ciencia. De la misma forma propone recuperar las exigencias que para la epistemología del sujeto cognoscente debe tener el conocimiento científico y la posibilidad de que el sujeto conocido sea al mismo tiempo una parte activa en la construcción cooperativa del conocimiento y una presencia no oscurecida o negada, sino integralmente respetada en la transmisión de este Vasilachis (2006).

\section{Conclusiones}

El diseño metodológico de la presente investigación combinará los métodos cuantitativos y cualitativos, pues debido a la intencionalidad de la investigación, es necesario establecer información en profundidad sobre aspectos subjetivos, percepciones y pensamientos de los grupos de estudio sobre el trabajo, que las técnicas de investigación etnográfica desarrollan y se complementan con los datos cuantitativos. En este propósito la epistemología del sujeto conocido de Vasilachis nos abre el espectro de comprensión para sugerir propuestas que se adapten a las realidades complejas de nuestras sociedades.

La implementación de la investigación cualitativa y el marco interdisciplinar que se aborda en la investigación nos facilitará aportar elementos para la comprensión del concepto de trabajo hoy en Colombia, que nos permita entender y plantear desde su percepción algunas soluciones a la crisis del empleo. Asimismo, comparar intergeneracionalmente las percepciones que tienen 
hoy sobre el trabajo los tres grupos focales, con el fin de construir propuestas afines y pertinentes a las necesidades laborales de los colombianos.

En un mundo cada vez más complejo, en el que es cada vez más difícil conseguir y mantenerse en el trabajo que se desea, debido, entre otras cosas, a la implementación de las medidas neoliberales, se hace necesario aportar soluciones de ocupación a los miles de jóvenes egresados y próximos a salir al mercado laboral, así como sugerir nuevas concepciones, posibilidades de trabajo y de desarrollo humano para la sociedad colombiana.

\section{Referencias}

Aigneren, Miguel. (2007). «La técnica de recolección de información mediante los grupos focales». Artículo publicado en CEO, Revista Electrónica. 7. Disponible en: http://huitoto.udea. edu.co/ ceo//www.elprisma.com/apuntes/curso.asp?id=2686.

Alperovitz, G. (1993, junio). «The Clintonomics Trap». The Progressive.

Antunes, R. Pochmann M. (2008). La desconstrucción del trabajo y la explosión del desempleo estructural y de la pobreza en Brasil, Disponible en: http://bibliotecavirtual.clacso.org.ar/ar/ libros/clacso/crop/cattani/08antu.pdf

Arenas, G. (1990). La contratación de trabajadores a través de empresas de servicios temporales, disponible en: www.icesi. edu.co/biblioteca_digital/bitstream/item/424/1/c4b-garenascontratacion_temporales.pdf

Arendt, H. (1998). La condición humana. Barcelona: Paidós.

Bauman, Z. (2000). Trabajo, consumismo y nuevos pobres. Barcelona: Gedisa.

Beck, U. (2008). ¿Qué es la globalización? Argentina: Paidos.

ITINERARIO EDUCATIVO • AÑO XXVI, N. ${ }^{\circ} 59 \cdot 53-78 \cdot$ ENERO - JUNIO DE ZOIL 
Bisquerra, R. (1990). Métodos de investigación educativa. Ceac Educación, Manuales.

Bonilla, C. E. \& Rodríguez, P. (1997). Más allá del dilema de los métodos. Bogotá: Norma.

Bronstein, A. (1990). «La flexibilidad del trabajo: Panorama general», en La flexibilización del trabajo. Un estudio internacional. Co. Óscar Hernández Álvarez. Barquisimeto: Instituto Latinoamericano de Derecho del Trabajo.

De la Garza. (2001). «Problemas clásicos y actuales de la crisis del trabajo». En C. Neffa y E. de la Garza Toledo (Comp.). El trabajo del futuro. El futuro del trabajo. Buenos Aires: CLACSO.

Denzin, N. K., Lincoln, Y. S. (1994). «Introduction: Entering the Field of Qualitative Research»y «The Fifth Moment». En Denzin, N. K., Lincoln (eds.). Handbook of Qualitative Research. California: Sage Publications.

Diario Portafolio. (Enero 23 de 2010).

Engels, F. (1979). El papel del trabajo en la transformación del mono en hombre, Cali: Andreus.

Fals Borda, O. (1990.) «El tercer mundo y la reorientación de las ciencias contemporáneas». Revista Nueva Sociedad. 180-181, 169-181.

Flick, U. (1998). An Introduction to Qualitative Research. Londres: Sage.

Garay, L. (2004). Colombia: estructura industrial e internacionalización 1967-1996, Biblioteca Virtual del Banco de la República, disponible en: www.banrepcultural.org/blaavirtual/economia/ industrilatina/indice.htm

Goetz y LeComte. (1988). Citado por Bisquerra Rafael en: Métodos de investigación educativa. 1990. CEAC Educación Manuales. 
Gorz, A. (1991). Metamorfosis del trabajo. Búsqueda de sentido. Crítica de la razón económica. Madrid: Sistema.

Gorz, A. (1998). Miseria del presente, riqueza de lo posible. Argentina: Paidós.

Jacob, A. (1995). La noción de trabajo. Relato de una aventura socio-antropo-histórica. Sociología del trabajo. Piette, disponible en línea en: www.ceil-piette.gov.ar/docpub/documentos/ sociologiadeltrabajo/st4jacob.pdf.

Jáuregui, F. (1996). El tiempo que vivimos. Barcelona: Paidós.

Marx, C. \& Engels, F. (1972). Trabajo asalariado y capital. Obras Escogidas. Moscú: Progreso.

Méda. D. (1995). El trabajo, un valor en peligro de extinción. Barcelona: Gedisa.

Osorio, R. (2001). El Cuestionario. Disponible en: www.nodo50. org/sindpitagoras/Likert.htm

Pérez, G. (2001a). Investigación cualitativa. Retos e interrogantes. Métodos. Madrid: La Muralla.

Portela, E. (2001). El cambio feudal. El hombre y la tierra. Señores y campesinos. En Claramunt, S. E., Portela, S., González y E. Mitre (2001). Historia de la Edad media. Barcelona: Arial.

Offe, C. (1985). «Le travail comne categorie de la sociologie», Les temps modernes. 466.

Quintana, A. (2006). «Metodología de Investigación Científica Cualitativa». Psicología: Tópicos de actualidad, Quintana, A. y Montgomery, W. (Eds.). Lima: UNMSM.

Rifkin, J. (2004). El fin del trabajo. España: Paidos.

Roediger, D., Phiplip, F. (1989). Our Uwn Time: A history of American Labor and the Working Day, Westport, CT, Greeenwood Press. 
Rosenman, S.I., (Comp.). (1938). The Puiblic Papers am Adress of Franklin D. Roosevelt. 2, Nueva York: The Yera fi Crisis, 1933.

Santa María, M., García, F., Mujica, A. (2009). Los costos no laborales y el mercado laboral: Impacto de la reforma de salud en Colombia, Working Paper, 43 de 2009-08, Fedesarrollo, disponible en: www.fedesarrollo.org.co/wp-content/ uploads/2011/08/WP-No.-43-Los-costos-no-salariales-y-elmercado-laboral.pdf

Sennett, R. (2006). La corrosión del carácter. Barcelona: Anagrama

Tirado A. (1981). Introducción a la historia económica de Colombia. Bogotá: La Carreta.

Turuguet, Doménech. (1992). Sobre el análisis documental. Recuperado el 24 de mayo de 2007 de: www.elprofesionaldelainformacion. com/contenidos/1992/noviembre/sobre_el_anlisis_documental.html.

Vasilachis, I. (2003). Pobres, pobreza, identidad y representaciones sociales. Barcelona: Gedisa.

Vasilachis, I. (2006). «La investigación cualitativa». Estrategias de Investigación Cualitativa, Coor, Vasilachis, I. Barcelona Gedisa.

Vera, L. (2004). La investigación cualitativa. Recuperado en mayo 27 de: http://ponce.inter.edu/cai/reserva/lvera/INVESTIGACION_CUALITATIVA.pdf

Weber, M. (2002). La ética protestante y el espíritu del capitalismo, Barcelona: Alianza.

Yunis, E. (2004). ¿Por qué somos así? Bogotá: Temis. 